Recepción: 20 / 04 / 2017

Aceptación: 20 / 05 / 2017

Publicación: 15 / 09 / 2017

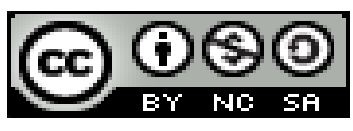

Ciencias sociales y políticas

Artículo Científico

\title{
Recurso hídrico, Clima y Sistemas de Información Geográfica
}

Water resources, Climate and Geographic Information Systems

Recursos hídricos, sistemas de informação climática e geográfica

Silvia Cargua ${ }^{\mathrm{I}}$ val_ecu@hotmail.es

Marlene Cueva II josethtvb@hotmail.com

Jessica Escobar III josethtvb@hotmail.com

Susana Arciniegas IV srarciniegas@uce.edu.ec

Edwin Dimitri Nieto Guerrero ${ }^{\mathrm{V}}$ ednieto@uce.edu.ec

Correspondencia: val_ecu@hotmail.es

I

Docente de la Universidad Central del Ecuador, Quito, Ecuador.

Docente de la Universidad Central del Ecuador, Quito, Ecuador.

Docente de la Universidad Central del Ecuador, Quito, Ecuador.

Docente de la Universidad Central del Ecuador, Quito, Ecuador.

Docente de la Universidad Central del Ecuador, Quito, Ecuador. 


\section{Resumen}

El estudio es en el cantón Cayambe, de la provincia de Pichincha del Ecuador, que se ha identificado como cluster florícola, producción de lácteos y cluster turístico. Todas estas actividades han provocado un crecimiento poblacional que está causando un incremento en el índice de contaminación ambiental por descargas líquidas y sólidas de origen doméstico y agropecuario a los ríos sin tratamiento, lo que indica una relación directa sociedad-ambiente, a lo que se suma cambios en el clima como son las precipitaciones (fenómenos atmosféricos) y la temperatura (factor ambiental), componentes que inciden en la dinámica del recurso hídrico, estableciendo una relación directa sociedad-salud-ambiente. El proyecto tiene como finalidad, conocer la variabilidad climática de la zona en base a los datos de precipitación, temperatura, humedad relativa, así como, calcular índices de erosión, déficit hídrico e índice ombrotérmico mediante la aplicación de Sistemas de Información Geográfica.

Primero, se realizó el cálculo de la pendiente. Luego, en base a los datos de las estaciones meteorológicas, se aplicó el modelo geoestadístico kriging ordinario de predicciones, para realizar la interpolación y así obtener un modelo de precipitación y temperatura del Cantón. Con los resultados se calculó el déficit hídrico, aplicando la herramienta "Algebra de Mapas", para finalmente calcular la "Erosión” y el "Índice Ombrotérmico" en todo el cantón.

El Índice Ombrotérmico, indicó que el Cantón Cayambe posee un clima lluvioso. Aunque, debido a varios factores que han contribuido al cambio climático, en los últimos 20 años, la zona presenta incremento en la temperatura, al igual que se han observado ligeras variaciones en la intensidad y frecuencia de la precipitación. Es decir, las variables calculadas permiten tomar atención a la disponibilidad hídrica durante la estación cálida.

Esto permitió definir una metodología que puede ser aplicada por el GAD (Gobierno Autónomo Descentralizado) en su propuesta de ordenamiento territorial.

Palabras claves: Modelización; algebra de mapas; kriging; recurso hídrico; clima; ombrotérmi. 


\section{Abstract}

The study is in Cayambe, in the province of Pichincha in Ecuador, which has been identified as a flower cluster, dairy production and tourist cluster. All these activities have led to a population growth that is causing an increase in the index of environmental pollution by liquid and solid discharges of domestic and agricultural origin to the rivers without treatment, indicating a direct relation society-environment, in addition changes in the climate such as precipitation (atmospheric phenomena) and temperature (environmental factor), components that influence the dynamics of the water resource, establishing a direct relationship between society and health. The project aims to know the climatic variability of the area based on precipitation, temperature and relative humidity data, as well as to calculate erosion indexes, water deficit and ombrothermal index through the application of Geographic Information Systems.

First, the slope calculation was performed. Then, based on meteorological station data, the ordinary prediction kriging geostatistics model was applied to perform the interpolation to obtain a precipitation and temperature model of the Canton. With the results the water deficit was calculated, applying the tool "Map Algebra", to finally calculate the "Erosion" and the "Ombrothermal Index" in the whole canton.

The Ombrothermal Index, indicated that the Cayambe Canton has a rainy climate. Although, due to several factors that have contributed to climate change, in the last 20 years the area has increased temperature, as have slight variations in the intensity and frequency of precipitation. That is, the calculated variables allow attention to water availability during the warm season.

This allowed defining a methodology that can be applied by the GAD (Decentralized Autonomous Government) in its proposal of territorial organization.

Key words: Modeling; map algebra; kriging; hidric resource; weather; ombrotérmi. 


\section{Introducción.}

San Pedro de Cayambe es una ciudad del noreste de la provincia de Pichincha en Ecuador. Es la cabecera del cantón Cayambe; toma su nombre del volcán Cayambe, al pie de cuya ladera occidental se asienta. Por esta ciudad atraviesa la línea Ecuatorial. Su población es de 39.028 habitantes, siendo la tercera ciudad más poblada de la provincia.

El estudio que se realizó en el cantón Cayambe tuvo como finalidad conocer el cambio climático de la zona que se ha dado en los últimos 30 años, para lo cual emplearemos datos de precipitación, temperatura, humedad relativa, entre otros, además mediante la utilización de sistemas de información geográfica (SIG) se determinaron los índices de erosión, déficit hídrico, índice ombrotérmico, zonas con mayor precipitación y zonas urbanas y rurales.

\section{Marco Teórico}

\section{Déficit hídrico}

Balance hídrico: "Es una representación teórica de los intercambios de agua entre las plantas, el suelo y la atmósfera” (Francisco Jiménez Otárola 1994).

El balance hídrico permite establecer las ganancias y pérdidas de agua que se registran en un área dada, por lo tanto se lo puede expresar en términos de:

$$
\text { Precipitación }+ \text { riego }=\text { evapotranspiración }+ \text { drenaje }+ \text { escurrimiento }
$$

No toda el agua que precipita se aprovecha y no siempre la frecuencia de las lluvias es constante, entre año tras año, por lo que se debe manejar los datos meteorológicos de 30 años 
mínimos para estimar la distribución. Otra variable a tomar en cuenta es la Tasa de infiltración de acuerdo a su textura.

\section{Zona de Humedad en un suelo}

La capa superficial freática se la conoce como zona de aireación o zona vadosa, en la cual la humedad puede distribuirse de tal manera que se distingan tres sub zonas

a) Sub zona de evapotranspiración: puede alcanzar desde centímetros si esta no está cubierta de vegetación hasta varios metros.

b) Sub zona capilar: en esta capa el agua ha ascendido por capilaridad a través de la porosidad del material.

c) Sub zona intermedia: se encuentra en medio de las dos sub zonas anteriores.

\section{Déficit Hídrico}

Se define como la escasez o pérdida de agua en un lugar determinado, que va ligado directamente con la evaporación y transpiración.

Evaporación: Es el fenómeno físico en el que el agua pasa de líquido a vapor y se produce de diferentes zonas:

- La superficie del suelo y la vegetación inmediatamente después de la precipitación

- Desde la superficie de agua (ríos, lagos, embalses)

- Desde el suelo, agua infiltrada que se evapora desde la parte más superficial del suelo. 
Transpiración: Es el fenómeno biológico por el que las plantas pierden agua a la atmósfera.

Para el análisis es de interés medir la cantidad total de agua que se pierde a la atmósfera por lo que se considera el cálculo de la Evapotranspiración (ET), siendo una de las aplicaciones el conocer las necesidades hídricas en campos de cultivo o en la zona para identificar la que mantenga mayor pérdida de agua por lo que a la ET se la conoce también como déficit de escorrentía o déficit hídrico.

Evapotranspiración: existen dos tipos de ET, la Evapotranspiración potencial (ETP) que es la producida si la humedad del suelo y la cobertura vegetal estuvieran en condiciones óptimas, y la Evapotranspiración real (ETR) que es la que se produce realmente en las condiciones de cada caso.

Si la ETR $\leq$ ETP se refiere a un lugar desértico, pero si la ETR=ETP se refiere a que la humedad del suelo es óptima y que existe un buen desarrollo vegetal.

\section{Factores que influyen al déficit hídrico}

Depende del poder evaporante de la atmósfera, que a su vez depende de los siguientes factores:

- Radiación solar

- Temperatura

- Humedad: menos humedad más evaporación.

- Presión atmosférica: a menor presión y mayor altitud más evaporación.

- Viento: más viento más evaporación 
Existen varios métodos para el cálculo de la ETP mes a mes para datos medios de una serie de años, con la Precipitación mensual se realiza un balance hidrológico mes a mes del agua en el suelo con lo que se obtiene la ETR, entonces.

Déficit hídrico $=E T P-E T R$.

Para el estudio, la formula (a) se reduce a

Déficit hídrico $=E T R$

\section{Análisis de la evapotranspiración (et)}

$$
E T R=\frac{P}{\sqrt{0,9+\frac{P^{2}}{L^{2}}}}
$$

- Donde:

- $\mathrm{ETR}=$ evapotranspiración real en $\mathrm{mm} / \mathrm{año}$

- $\mathrm{P}=$ precipitación en $\mathrm{mm} / \mathrm{año}$

- $\mathrm{L}=300+25 \mathrm{t}+0,5 \mathrm{t}^{3}$

- $\mathrm{t}=$ temperatura anual en ${ }^{\circ} \mathrm{C}$

\section{Erosión del Suelo}

La erosión del suelo se define como la pérdida de sus propiedades físicas y químicas, ya sea por factores antrópicos o naturales. Existen dos tipos de erosión: erosión hídrica y erosión eólica. 
Este trabajo se centrará en la erosión hídrica presente en el Cantón Cayambe. La erosión hídrica o erosión del suelo por escurrimiento hídrico tiene su origen en la acción del agua sobre una superficie desprovista de cobertura vegetal, según (Honorato et al., 2001) este proceso es quizás el más importante de degradación de suelos, dado que es irreversible y generalmente de gran magnitud.

Para determinar la erosión del suelo perteneciente al cantón Cayambe se va a emplear la Ecuación Universal de la Pérdida del Suelo; la misma que proporciona un cálculo de la media de la pérdida anual de suelo de tierras arables bajo diversas condiciones de cultivo. La aplicación de este cálculo tiene por objeto dar a los agricultores y a los técnicos en conservación de suelos la posibilidad de elegir combinaciones de usos de la tierra, prácticas de cultivo y prácticas de conservación del suelo que mantengan la pérdida de suelo a un nivel aceptable; con la terminología actual se diría que tiene por objeto lograr que el sistema agrícola sea sostenible. Como se concibió para ser usada en el campo tenía que ser "fácil de resolver e incluir sólo factores cuyo valor en un lugar particular se pueda determinar a partir de los datos disponibles. Algunos detalles y perfeccionamientos posibles se sacrificaron en aras de la utilidad" (Wischmeier 1976).

$A=R * K * L * S * C$

Dónde:

A es la media de la pérdida anual de suelo en toneladas por hectárea. (ton/ha/año)

$\mathrm{R}$ es una medida de las fuerzas erosivas de las precipitaciones y la escorrentía. (mm) 
$\mathrm{K}$ es el factor de erosionabilidad del suelo, es decir, una cifra que refleja la susceptibilidad de un tipo de suelo a la erosión o sea la recíproca de la resistencia del suelo a la erosión. Se mide en MJ*mm/ha*h

L es el factor de longitud, una relación que compara la pérdida de suelo con la de un campo de una longitud específica de 22,6 metros. (adimensional)

$\mathrm{S}$ es el factor de manejo, relación que compara la pérdida de suelo con la de un campo de pendiente específica del 9\%. (adimensional)

$\mathrm{C}$ es un factor de manejo de los cultivos, relación que compara la pérdida de suelo con la de un campo sometido a un tratamiento estándar de barbecho. (adimensional)

\section{Factor $\boldsymbol{R}$}

El factor R es el factor de lluvia en forma de un índice EI30, que es medido por el poder erosivo de la lluvia, una medida de las fuerzas erosivas de la lluvia y escurrimiento asociado.

$R=\frac{I_{30}(9.28 \mathrm{P}-8383)}{1000}$

Donde:

$\mathrm{R}=$ Factor de erosividad de las lluvias $\left(\mathrm{MJ}^{*} \mathrm{~mm} / \mathrm{ha} * \mathrm{~h}\right)$

$\mathrm{I} 30=75 \mathrm{~mm} / \mathrm{h}$ (valor recomendado por Wischmeier).

$\mathrm{P}=$ Precipitación promedio anual en $\mathrm{mm}$. 
RAMÍREZ (2010) señala que, el índice EI30, se define como el producto de la energía cinética (E) de un aguacero y su máxima intensidad en un intervalo de 30 minutos (I). El factor R resulta de promediar totales anuales de $\mathrm{E}^{*} \mathrm{I}$ para un período de por lo menos 30 años de fajas pluviográficas.

\section{Factor L}

Es adimensional. Donde, $\mathrm{m}$ es el exponente de la longitud de la pendiente y $\beta$ es el ángulo de la pendiente. La longitud de la pendiente se define como la distancia horizontal desde donde se origina el flujo superficial al punto donde comienza la deposición o donde la escorrentía fluye a un canal definido (Foster et al., 1977, citado por BARRIOS y QUIÑONEZ, 2000)

$$
m=\frac{F}{(1+F)} \quad F=\frac{\sin \beta / 0,0896}{3(\sin \beta)^{0,8}+0,56}
$$

El factor L con el área de drenaje aportadora (Desmet \& Govers, 1996, citado por VELÁSQUEZ, 2008).

$$
L_{(i, j)}=\frac{\left(A_{(i, j)}+D^{2}\right)^{m+1}-A_{(i, j)}{ }^{m+1}}{x^{m} \cdot D^{m+2} \cdot(22,13)^{m}}
$$

Donde A $(i, j)[m]$ es el área aportadora unitaria a la entrada de un pixel (celda), D es el tamaño del pixel y x es el factor de corrección de forma.

\section{Factor $\mathbf{S}$}

El ángulo $\beta$ se toma como el ángulo medio a todos los subgrids en la dirección de mayor pendiente (McCOOL et al., 1987,1989, citado por BARRIOS y QUINONEZ, 2000). 
Silvia Cargua; Marlene Cueva; Jessica Escobar; Susana Arciniegas; Edwin Dimitri Nieto Guerrero

$$
S_{(i, j)}= \begin{cases}10,8 \sin \beta_{(i, j)}+0,03 & \tan \beta_{(i, j)}<0,09 \\ 16,8 \sin \beta_{(i, j)}-0,5 & \tan \beta_{(i, j)} \geq 0,09\end{cases}
$$

VELÁSQUEZ (2008) nos dice que, cuando se aplica esta fórmula en el Raster Calculator de ArcGIS se debe tomar en cuenta que el ángulo deberá ser convertido a radianes (1 grado sexagesimal $=0,01745$ radianes), para que pueda ser multiplicado por los demás componentes de las ecuaciones. Es adimensional.

\section{Factor $\boldsymbol{K}$}

Es el factor de erodabilidad del suelo, es la erosión estándar en tonelada por hectárea por unidad de erosividad $\mathrm{R}$, para un suelo específico con una pendiente uniforme y una longitud de pendiente en barbecho limpio labrado. Es una medida de la susceptibilidad inherente de las partículas del suelo a la erosión. Se mide en (ton*ha*h/MJ*mm*ha)

\begin{tabular}{|l|c|c|c|}
\cline { 2 - 4 } \multirow{2}{*}{ Textura } & \multicolumn{3}{|c|}{$\%$ de materia orgánica } \\
\cline { 2 - 4 } & $\mathbf{0 . 0 - 0 . 5}$ & $\mathbf{0 . 5}-\mathbf{2 . 0}$ & $\mathbf{2 . 0 - 4 . 0}$ \\
\hline Arcillo arenosa & 0.014 & 0.013 & 0.012 \\
\hline Arcillo limosa & 0.025 & 0.023 & 0.019 \\
\hline Arena & 0.005 & 0.003 & 0.002 \\
\hline Arena fina & 0.016 & 0.014 & 0.010 \\
\hline Arena fina migajosa & 0.024 & 0.020 & 0.016 \\
\hline Arena migajosa & 0.012 & 0.010 & 0.008 \\
\hline Arena muy fina & 0.042 & 0.036 & 0.028 \\
\hline Arena muy fina migajosa & 0.044 & 0.038 & 0.030 \\
\hline Limo & 0.060 & 0.052 & 0.042 \\
\hline Migajón & 0.038 & 0.034 & 0.029 \\
\hline Migajón arcillo arenosa & 0.027 & 0.025 & 0.021 \\
\hline Migajón arcillo limosa & 0.037 & 0.032 & 0.026 \\
\hline Migajón arcillosa & 0.028 & 0.025 & 0.021 \\
\hline Migajón arenosa & 0.027 & 0.024 & 0.019 \\
\hline Migajón arenosa fina & 0.035 & 0.030 & 0.024 \\
\hline Migajón arenosa muy fina & 0.047 & 0.041 & 0.033 \\
\hline Migajón limoso & 0.048 & 0.042 & 0.033 \\
\hline Arcilla & \multicolumn{3}{|c}{$0.013-.029$} \\
\hline
\end{tabular}

Cuadro 1: Valores de erosionabilidad de los suelos (K) estimado en función de la textura y el contenido de materia orgánica (Morgan 1985). 


\section{Índice ombrotérmico}

Cociente de dividir el valor de la precipitación positiva anual $(\mathrm{Pp})$; es decir, el sumatorio de la precipitación mensual en milímetros de los meses de temperatura media superior a cero grados centígrados y la temperatura positiva anual (Tp); es decir, el sumatorio de la temperatura mensual en décimas de grados centígrados de los meses de temperatura media superior a cero grados centígrados. Su fórmula es:

$$
I o=\frac{P p}{T p}
$$

Donde:

Io $=$ Índice ombrotérmico $\left(\mathrm{mm} /{ }^{\circ} \mathrm{C}\right)$

\section{Climodiagrama de Walter y Gaussen (diagrama ombrotérmico):}

La escala de precipitación debe ser el doble que la de temperatura. Cuando la precipitación es inferior a 2 veces la temperatura media en ${ }^{\circ} \mathrm{C}$ el mes se considera árido, si la precipitación es superior a este valor, el mes se considera semi húmedo y húmedo si la precipitación supera en tres veces a la temperatura media mensual.

Índices Ombrotérmicos según Rivas Martínez (2005)

Son un conjunto de índices que el autor usa para la clasificación de los bioclimas del mundo y que define como:

Índice ombrotérmico anual (Io), es el cociente entre la suma de la precipitación media en mm de los meses cuya temperatura media es superior a cero grados centígrados ( $\mathrm{Pp}$ ) y la suma de las temperaturas medias mensuales superiores a cero grados centígrados en décimas de grado (Tp). 


$$
I o=\frac{P p}{T p}
$$

"Estos índices indican que a escala global existen cinco macrobioclimas: Tropical, Mediterráneo, Templado, Boreal y Polar [30] (Rivas-Martínez 2008)”.

El Ecuador continental está contenido solamente en el macrobioclima Tropical. Dentro de cada macrobioclima se distinguen varios bioclimas, ombrotipos y termoclimas con intervalos de variación más pequeños, los cuales permiten caracterizar el clima de maneras cada vez más precisas. En la presente propuesta de clasificación, macrobioclima, bioclima y ombrotipo son factores de diagnóstico utilizados en los niveles IVC de Subclase, Formación y Ecosistema, respectivamente.

“Los análisis bioclimáticos realizados para la elaboración del Mapa de Ecosistemas de los Andes del Norte y Centro (Josse et al.2009)"; indican que en el Ecuador continental existen cuatro bioclimas y siete ombrotipos (Tabla 1).

\begin{tabular}{|c|c|c|}
\hline Bioclima & 10 & $\operatorname{lod} 2$ \\
\hline Desértico & $0,2-1,0$ & . \\
\hline Xerico & $1,0.3 .6$ & . \\
\hline Pluviestacional & $\geq 3,6$ & $\leq 0,7$ \\
\hline Pluvial & 23.6 & $>0,7$ \\
\hline Ombrotipo & 10 & \\
\hline Deserfico & 0.1 & \\
\hline Semi-trido Inferiox & $1 \cdot 1,5$ & \\
\hline Semi-arido superior & $1,5-2$ & \\
\hline Seco Inferiox & $2-2,8$ & \\
\hline $\begin{array}{l}\text { Seco Superior } \\
\text { Sub-humedo Inferior }\end{array}$ & $2,8-3,6$ & \\
\hline Suchumedo Superior & $4,8-7$ & \\
\hline Húmedo Inferior & $7-10,5$ & \\
\hline Humedo Superior & $10,5 \cdot 14$ & \\
\hline Hiper-hümedoinferior & & \\
\hline $\begin{array}{l}\text { Hiper-humedo } \\
\text { Superior }\end{array}$ & $\begin{array}{c}14-21 \\
21-28\end{array}$ & \\
\hline Utra-hiperhúmedo & $\geq 28$ & \\
\hline
\end{tabular}

Tabla 1: Bioclimas y ombrotipos presentes en el Ecuador continental

(Rivas-Martínez, 2008)

\section{Metodología}


El cambio climático está evidenciado por la alteración del comportamiento de las variables climáticas en un determinado lugar, por lo tanto, para determinar si este fenómeno está ocurriendo en el cantón Cayambe es necesario determinar y calcular las variaciones de estas variables con datos de un determinado rango de tiempo, en este caso será de 30 años.

\section{Mapa de pendiente del cantón Cayambe}

- Se calcula el mapa de pendiente (fig. 1).

- Se creó una tabla en la cual el valor de la pendiente media será: MEAN, valor en porcentaje

MAPA DE PENDIENTES

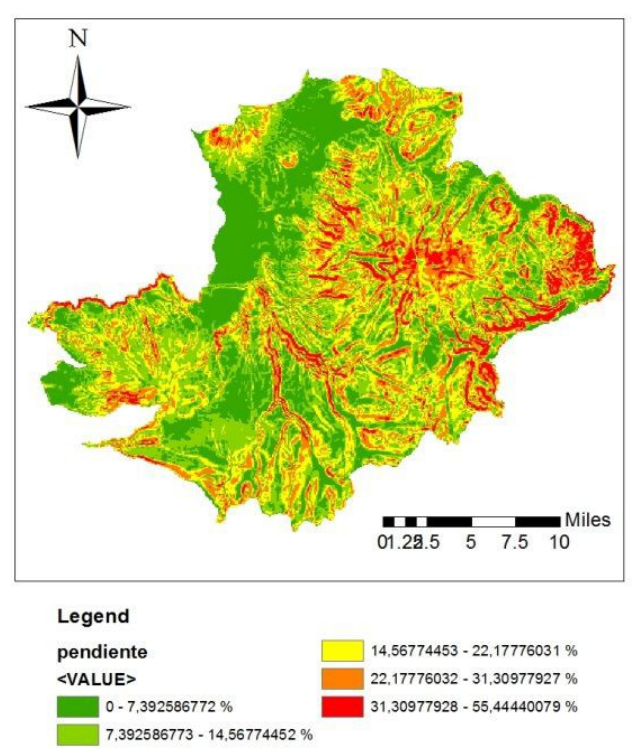

Figura 1. Mapa de Pendiente del Cantón Cayambe

\section{Mapa de precipitación del cantón Cayambe}

Para realizar el cálculo de la precipitación media se procedió con los siguientes pasos: 
- Recolectar la mayor cantidad de datos de precipitaciones disponibles del INAMHI que cubran el cantón Cayambe.

- A aquellos meses de las estaciones que les falte un máximo de dos datos se pueden llenar por PROMEDIO; pero a aquellos meses que les falten más de tres datos se deben rellenar mediante correlación.

- Para el relleno de correlación se procedió a llenar los datos faltantes; utilizando la estación que tiene sus datos completos y que servirá como base, la comparamos con los datos de la estación que se desea rellenar; es decir, se rellenaron mes a mes cada uno de los valores faltantes de precipitaciones para cada estación.

- Con el fin de mejorar u obtener un mejor $\mathbf{r}$ se aplicó estimación lineal.

- El proceso de estimación para rellenar los datos consiste en:

Hacer uso de los datos de la estación tomada como base (eje x) en función con la estación a ser rellenada (eje y), se realiza la estimación lineal. No hay que tomar en cuenta los datos faltantes. Esta estimación lineal nos arroja una ecuación y un coeficiente de correlación (este coeficiente deber ser mayor o igual a 0.75 ). Este procedimiento se lo debe realizar para cada mes.

- Una vez completa la información y validada se procede a calcular los parámetros estadísticos de cada estación que son: suma, media, valor máximo, valor mínimo.

- Para validar la información, después del relleno de los datos faltantes se elaboró la curva de Doble Masa; la cual consiste en graficar las sumatorias anuales acumuladas de los datos de precipitación de las estaciones rellenadas, Se comprobará si la información está bien rellenada si el grafico generado presenta una tendencia lineal con un coeficiente $\mathrm{R}$ aproximadamente igual a 1. 
Cálculo de la precipitación media

Para calcular la precipitación media en el mapa del Cantón Cayambe se elaboró una tabla de Excel con los siguientes elementos:

Código

Nombre de la Estación

Coordenada X (Long)

Coordenada Y (Lat)

Precipitación Media Multianual

Utilizando la herramienta de Sistemas de Información Geográfica se procedió a elaborar un plano raster de precipitación mediante la opción Kriging del Geostatistical Analyst Tools, posteriormente se convirtió en vector y finalmente a raster. 


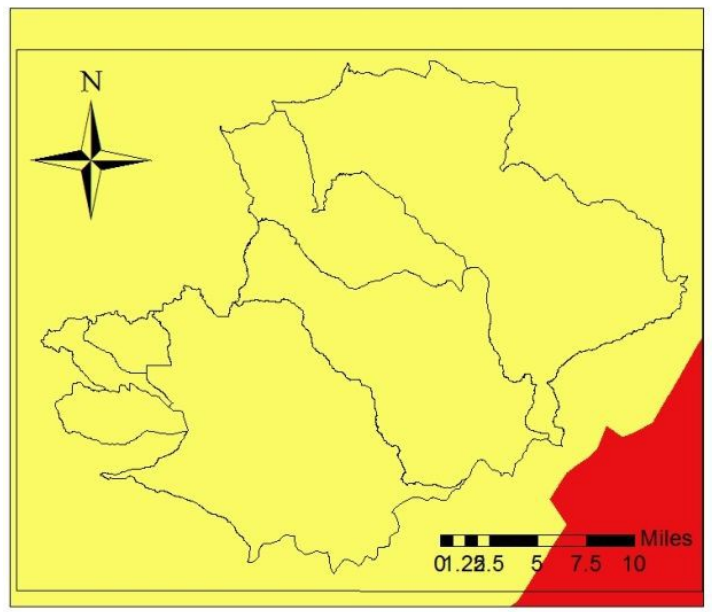

Legend

\begin{tabular}{l|l} 
Precipitación & Filled Contours \\
Prediction Map & $763.649908-1,003.04878 \mathrm{~mm}$ \\
\hline [precipitacion].[Precipitac] & $1,003.04878-1,707.53435 \mathrm{~mm}$
\end{tabular}

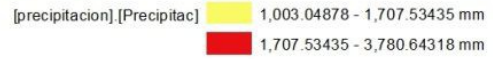

Figura 2. Precipitación del Cantón Cayambe

\section{Mapa de temperatura del cantón Cayambe}

- Recopilar toda la información disponible sobre la temperatura del Cantón Cayambe, la cual fue proporcionada por el INAMHI

- Rellenar los datos faltantes por promedio (máximo hasta dos datos).

- Para las estaciones de precipitación que no poseen temperatura, se las rellenó por correlación anual con las temperaturas medias de las estaciones llenas.

- Elaborar una tabla que contenga los datos de temperatura media de cada estación y exportarla a la herramienta de Sistemas de Información Geográfica.

○ Nombre

- Temperatura media multianual

○ Elevación 
- Coordenadas geográficas.

- Crear un Raster en función de la temperatura (Kriging) como se indicó en la parte de precipitación, cabe recalcar que se requirió la creación de Puntos auxiliares y con la herramienta Zonal Statistics se calculó la Temperatura media del Cantón Cayambe. Se debe repetir el proceso que se realizó para el mapa de precipitación.

\section{Déficit Hídrico}

Déficit hídrico $=E T P-E T R$

Cálculo de la evapotranspiración real (ETR), mediante el método de TURC

$$
E T R=\frac{P}{\sqrt{0,9+\frac{P^{2}}{L^{2}}}}
$$

Donde:

$\mathrm{ETR}=$ evapotranspiración real en mm/año

$\mathrm{P}=$ precipitación en $\mathrm{mm} / \mathrm{año}$

$\mathrm{L}=300+25 \mathrm{t}+0,5 \mathrm{t}^{3}$

$\mathrm{t}=$ temperatura anual en ${ }^{\circ} \mathrm{C}$

Estas estaciones están ubicadas en sectores agrícolas representativos de la zona. 


\section{DEFICIT HIDRICO}
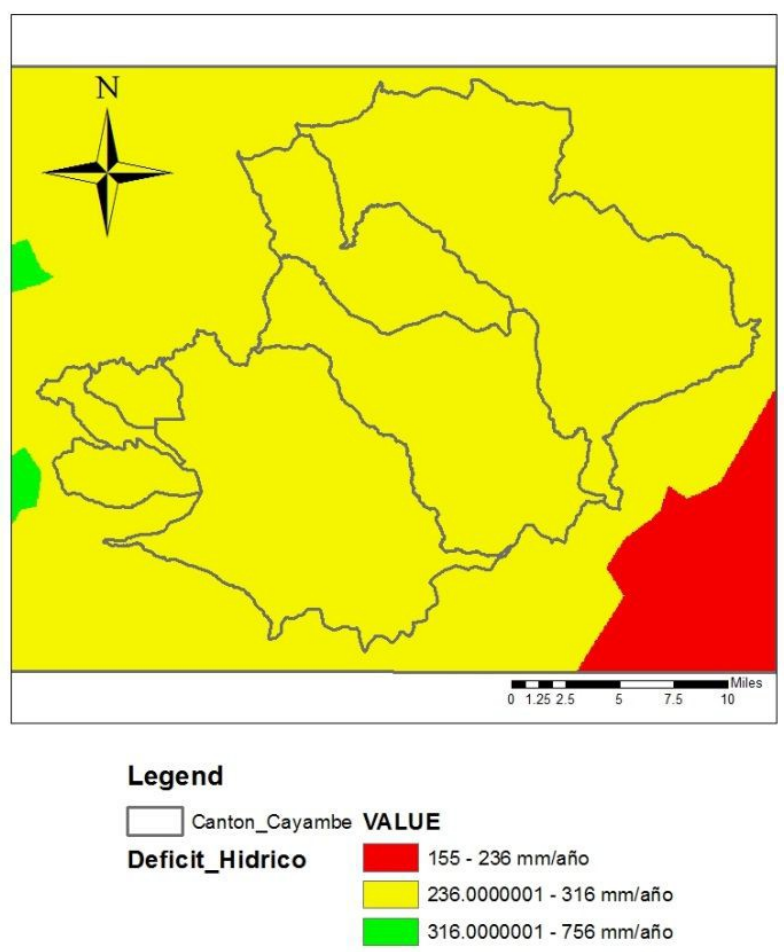

Figura 3. Déficit Hídrico

\section{Erosión del Suelo}

En función del modelo digital del terreno (MDT) se calculó el de pendiente, para crear un mapa con la dirección de flujo y la acumulación de flujo.

\section{Factor R}

Una vez realizado el kriging de precipitación se procede a exportarlo como vector, geometría de polígono, se lo clasifica de manera que estos valores incluyan a los mínimos (figura 4)

\section{Temperatura}


Se realiza el mismo procedimiento descrito para el caso de precipitación.

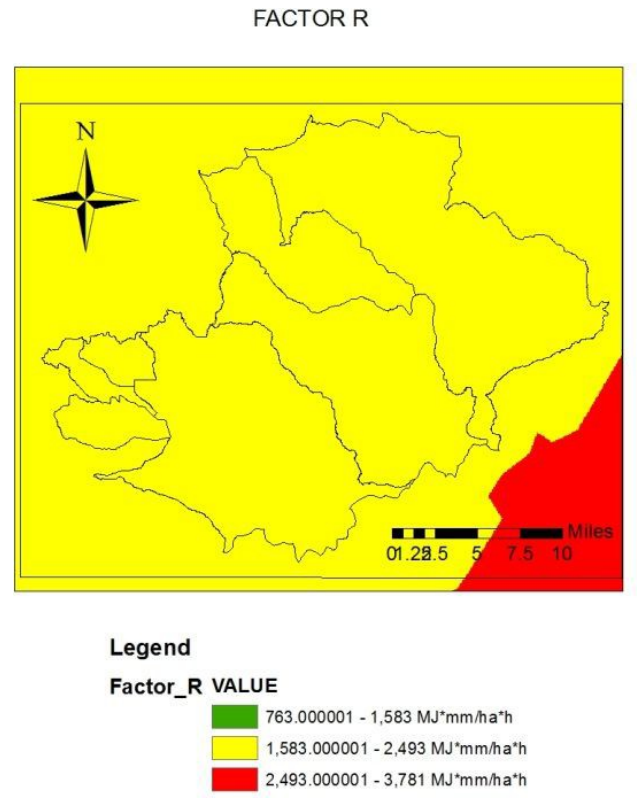

Figura 4. Factor $R$

\section{Factor $\mathbf{L}$}

Para realizar el cálculo de este factor se debe emplear la herramienta Algebra de Mapas.

- Calcular el factor F con la fórmula:

((Sin (“Slope”*0.01745)/0.0896)/(3*Power(Sin(“Slope”*0.01745),0.8))+0.56)) 
Silvia Cargua; Marlene Cueva; Jessica Escobar; Susana Arciniegas; Edwin Dimitri Nieto Guerrero

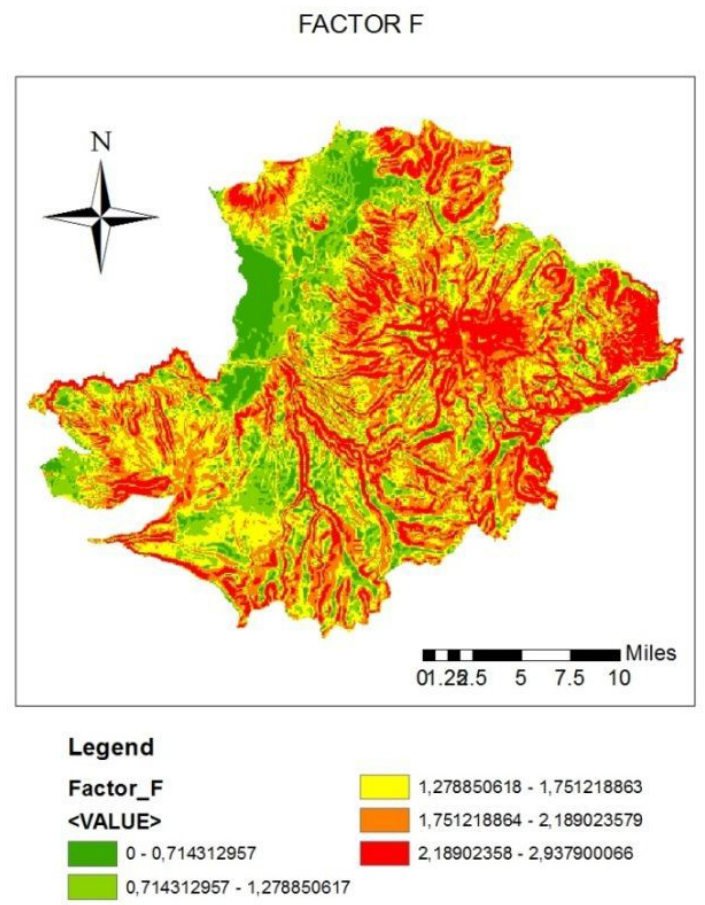

Figura 5. Factor $\boldsymbol{F}$

- Luego se calcula el factor m con la capa antes creada, con la fórmula:

"Factor_F"/ (1+"Factor_F") 


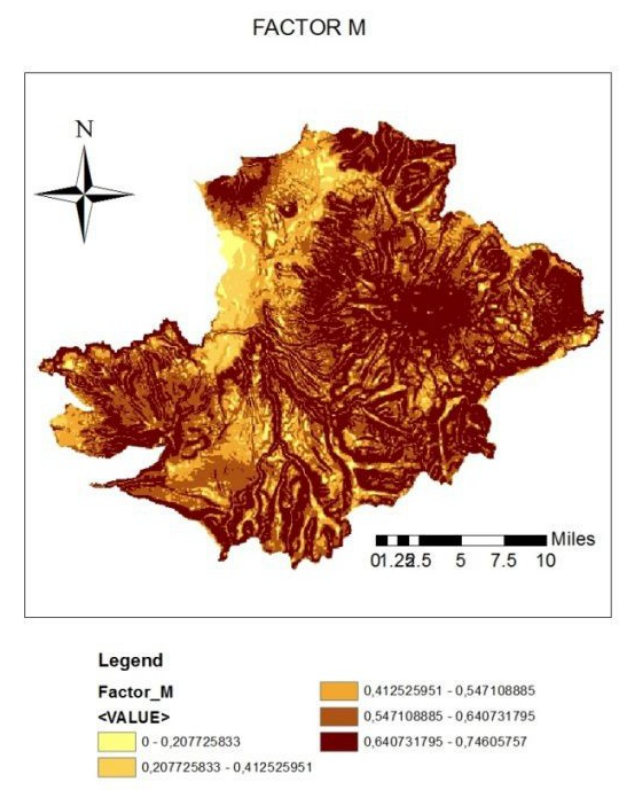

Figura 6. Factor M

- El cálculo del factor L requiere de la capa m, como muestra la fórmula en la parte de arriba; sin embargo, para utilizar el álgebra de mapas la ecuación va de la siguiente manera:

(Power ((“FlowAcc”+625),(“Factor_M”+1)) - Power(“FlowAcc”,(“Factor_M”+1) )) / (Power (25, (“Factor_M"+2*Power (22.13, "Factor_M")) 
Silvia Cargua; Marlene Cueva; Jessica Escobar; Susana Arciniegas; Edwin Dimitri Nieto Guerrero

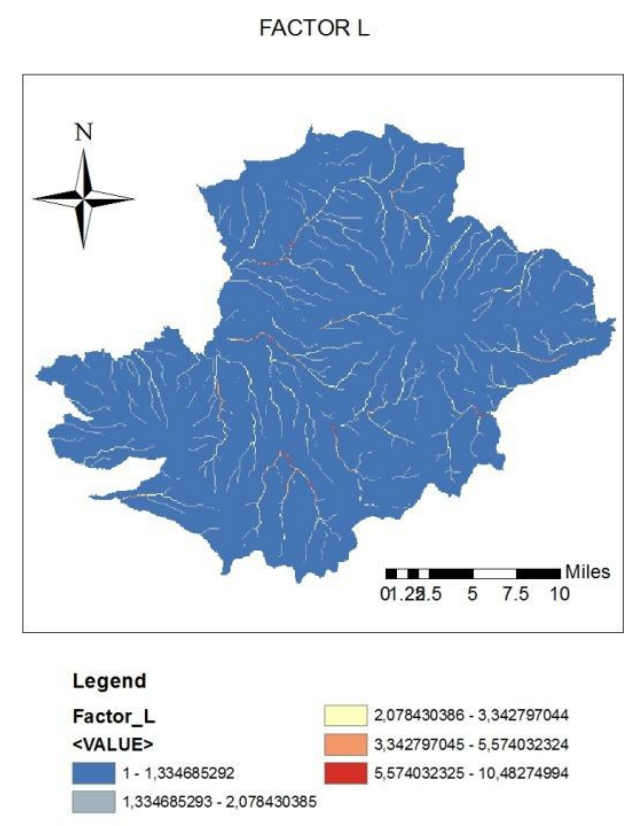

Figura 7. Factor $L$

\section{Factor S}

- El factor S se calcula con la siguiente fórmula en el Raster Calculator de Arcgis:

Con ((Tan ("Slope"*0,01745) < 0,09), (10,08* Sin ("Slope"0,01745)+0.03), (16,8*Sin ("Slope"

$* 0,01745)-0,5))$ 


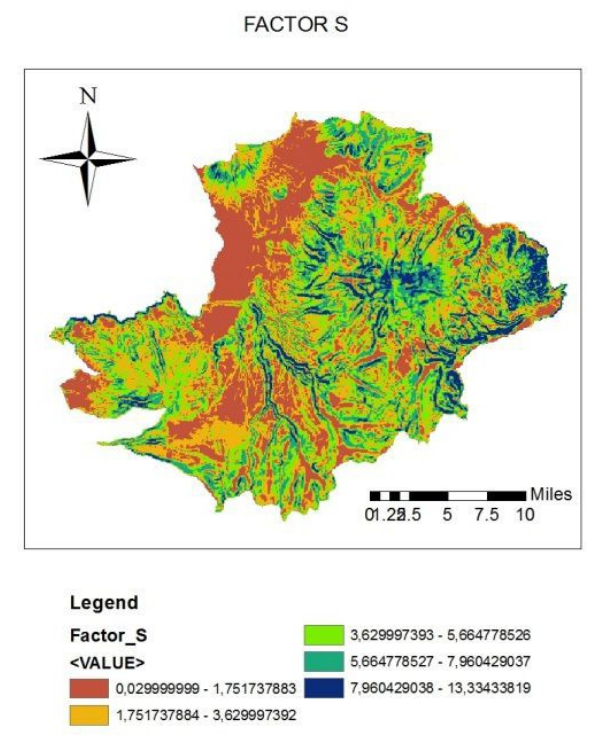

Figura 8. Factor $S$

\section{Factor LS}

El factor LS se calcula con la siguiente fórmula en el Raster Calculator "Factor_L" * "Factor_S"

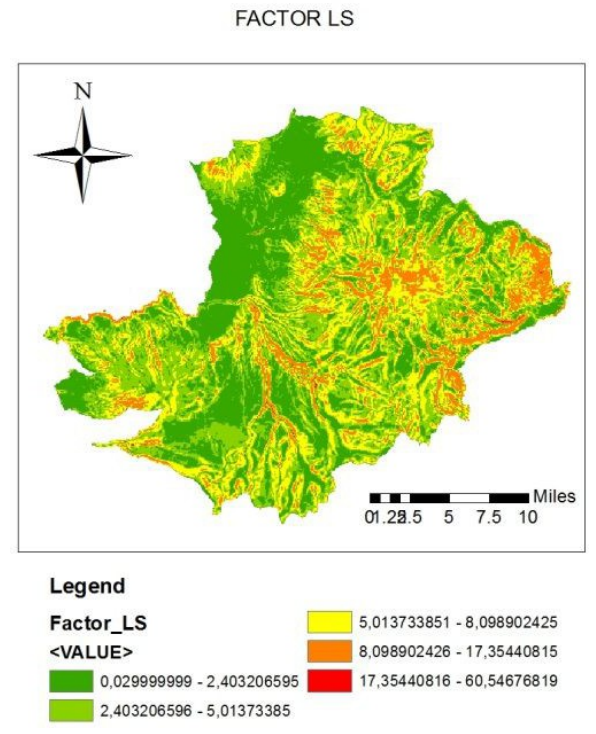

Figura 9. Factor LS 
Silvia Cargua; Marlene Cueva; Jessica Escobar; Susana Arciniegas; Edwin Dimitri Nieto Guerrero

\section{Factor K}

Para el cálculo de este factor es necesario conocer la edafología de la zona. Sin embargo, para el presente estudio se empleó el mapa de Taxonomía de todo el Ecuador y se lo comparó con la clasificación de la Soil Taxonomy y la U para de esta manera encontrar la textura del suelo.

Con la textura de cada subgrupo taxonómico se procedió a buscar los valores de la erodabilidad en la tabla que se muestra en la parte teórica del documento. En esta tabla el factor K se encuentra en función de la textura y el porcentaje de materia orgánica, que para efectos de este estudio se supuso de 0,5 a $2 \%$.

Colocarla en varios puntos y así crear otra capa con IDW, especificando un tamaño de celda de 34.

\section{FACTOR K}
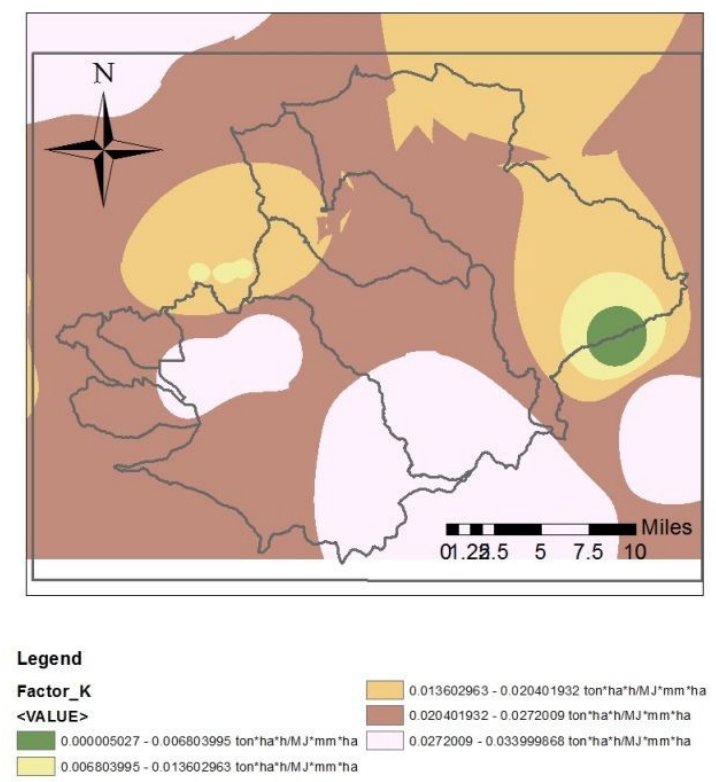

Figura 10. Factor $K$

\section{Factor C}


- Para este factor se necesita el mapa de los usos del suelo de la zona en cuestión, o el mapa de la cobertura vegetal. Para este estudio se procedió con el mapa de uso de suelos y cobertura del Ecuador. Luego se rellenaron los valores de C en relación con la cobertura vegetal, para ello se tomaron en cuenta tablas de varios autores ya que presentaban diferente nivel de especificación de la cobertura vegetal.

- Transformar de polígono a raster, el mapa y se consigue el Factor C.

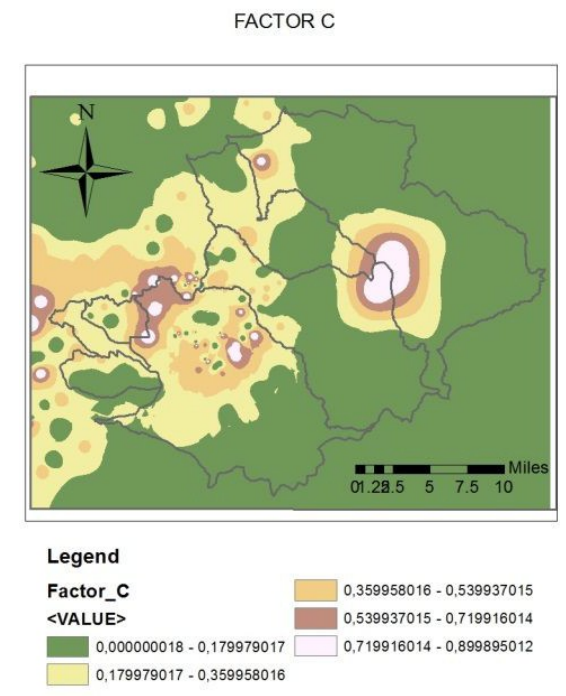

Figura 11. Factor C

\section{Factor A}

Finalmente, para calcular la erosión del suelo se debe aplicar la siguiente fórmula en el Raster Calculator:

"Factor_R"*”Factor_K"*”Factor_LS"*”Factor_C"

Nota: En cada capa se realiza una clasificación en las clases que se consideren necesarias, recomendable de 5 a 10, y cambiar los colores para una mejor observación. 
Silvia Cargua; Marlene Cueva; Jessica Escobar; Susana Arciniegas; Edwin Dimitri Nieto Guerrero

EROSION DEL SUELO

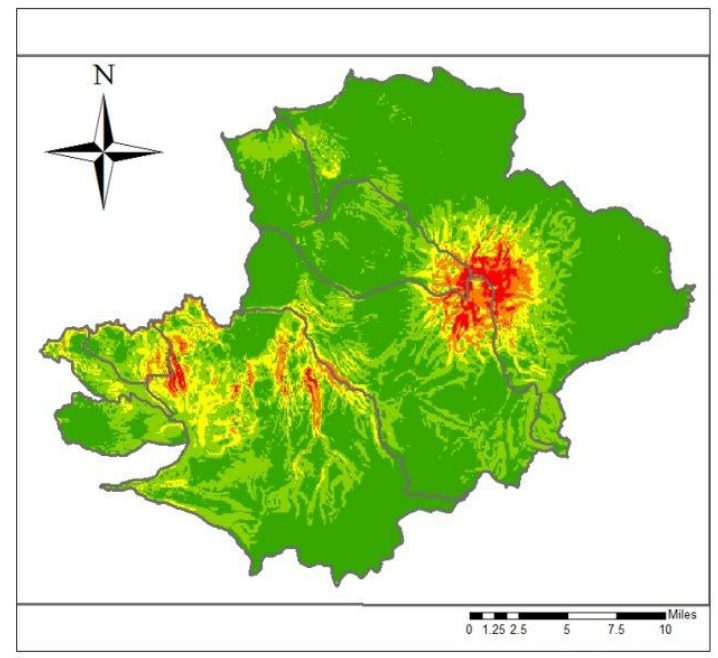

Legend

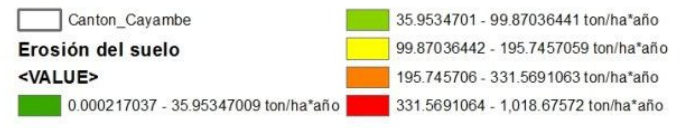

Figura 12. Factor A-Mapa de erosión del suelo en Cayambe

\section{Índice Ombrotérmico}

Se realizó una tabla de atributos con datos de precipitación y temperatura en Excel y se la trasladó a ARCGIS, posteriormente se utilizó la herramienta kriging para obtener el mapa del Índice Ombrotérmico. 
INDICE OMBROTERMICO

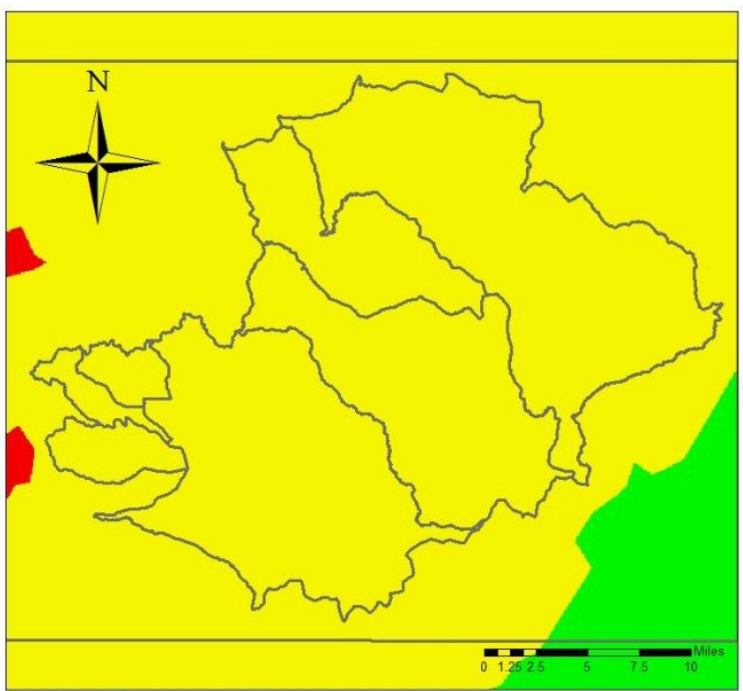

Legend

$$
\begin{aligned}
& \square \text { Canton_Cayambe <VALUE> } \\
& \text { Indice_Ombrotérmico } 3.039541721-6.400038663 \mathrm{~mm} /{ }^{\circ} \mathrm{C} \\
& 6.400038664-11.4102341 \mathrm{~mm} /{ }^{\circ} \mathrm{C} \\
& 11.41023411-18.5589276 \mathrm{~mm} /{ }^{\circ} \mathrm{C}
\end{aligned}
$$

Figura 13. Índice Ombrotérmico 


\section{Análisis de resultados}

\section{Déficit Hídrico}

Se define los rangos de déficit hídrico entre 155-236 mm como el nivel bajo, el 236-316mm

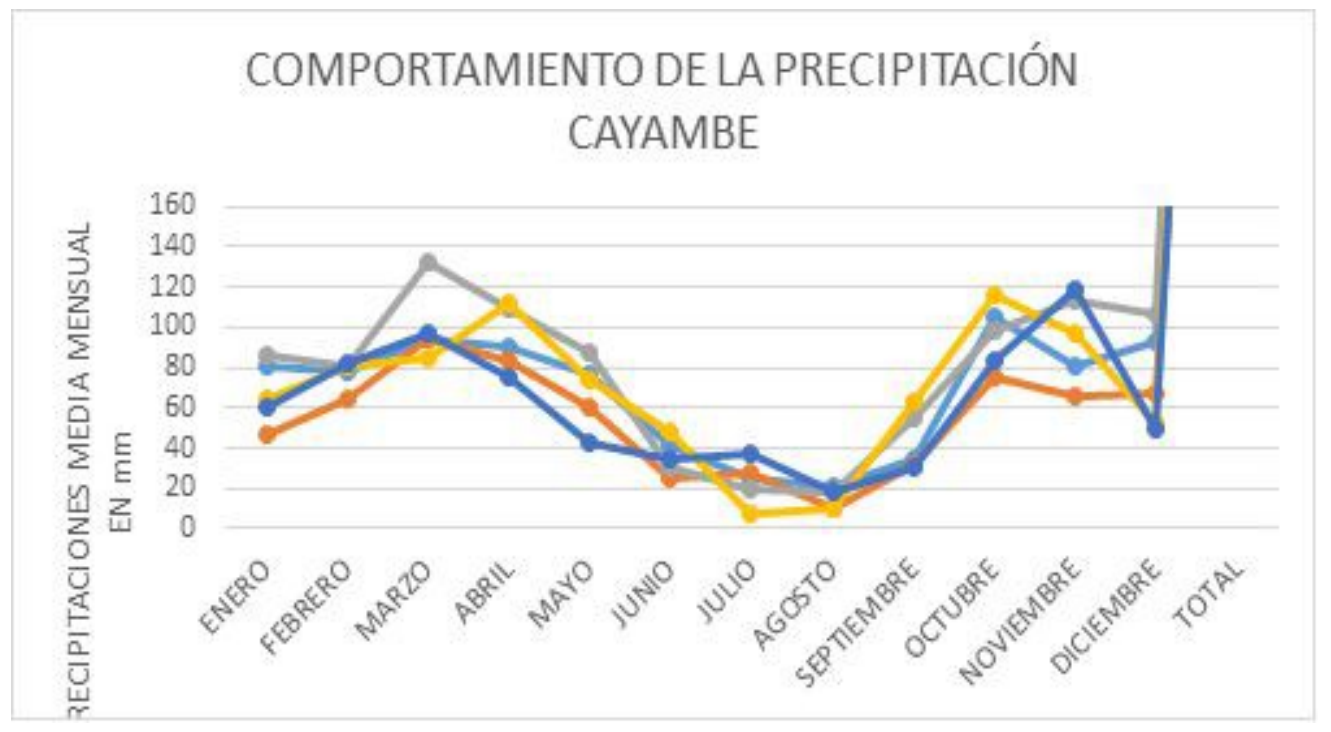

Figura 14: Comportamiento de la precipitación

Los meses de febrero, marzo y abril son los que presentan el mayor valor de temperatura, mientras que los meses de agosto y septiembre son los que presentan valores ligeramente más bajos con respecto a la media anual. Las variaciones mensuales de las temperaturas no son significativas ya que su amplitud (diferencia entre los valores máximos y mínimos) está alrededor de $2^{\circ} \mathrm{C}$.

Conociendo que la temperatura disminuye con la altura, en base a las curvas de nivel y mediante la ecuación establecida, se realizó el trazo de las isotermas que tienen valores entre 24 y $25^{\circ} \mathrm{C}$ a lo largo de todo el cantón, en general la variación de ETR, es desde 155mm a 756mm.

Dado que por éste método de cálculo se tomó en cuenta la temperatura media mensual, los valores de demanda atmosférica más elevados corresponden a los meses con mayor precipitación y los más bajos valores de ETR a los meses con menor humedad, acorde con los registros térmicos estacionales en el área. 


\section{Para la evaluación de cada una de las áreas.}

\begin{tabular}{|l|l|l|}
\hline \multirow{2}{*}{ ESTACIONES } & PERIODO HUMEDO & PERIODO SUBHÚMEDO \\
\hline \multirow{3}{*}{ CAYAMBE } & JULIO-OCTUBRE & $\begin{array}{l}\text { NOVIEMBRE } \\
\text { SIGUIENTE AÑO MAYO }\end{array}$ \\
\cline { 2 - 3 } & $\begin{array}{l}\text { AGOSTO } \\
\text { SEPTIEMBRE }\end{array}$ & CASI TODO EL AÑO \\
\hline
\end{tabular}

Evaluación desde 316 a 756

En este tipo de zonas se considera como las zonas de flujo de lava, ubicadas en pendientes de $5 \%$ hasta $100 \%$ y en donde la agresividad pluvial no sobrepasa los $100 \mathrm{~mm}$, las tierras van de poco profundas a profundas con texturas arenosas y franco limosos, generalmente usadas en prácticas agrícolas, pecuarias, gran parte por pastos cultivados,

Evaluación desde 236 a 316

En este tipo de zonas se considera las formaciones geológicas Aluvial cuaternario (relieves de las cuencas interandinas de clatos subredondeados), se distribuye en alrededor del $30 \%$ del cantón en la parte central y occidental del cantón las tierras van de superficiales a profundas con texturas de franco arenosos, franco arcilloso arenosos utilizadas para prácticas agrícolas, agropecuarias mixtas, cultivos de cebollas y cereales.

\section{Evaluación desde 155 a 236}

Es este tipo de zonas considerada las formaciones geológicas Cuaternaria (relieves flancos volcánicos y piroclásticos) con pendientes desde medias a fuertes, con una agresividad pluvial hasta los $100 \mathrm{~mm}$, en este tipo de zona la cobertura es pino, eucalipto, arbusto seco, con \{áreas en proceso de erosión y otras ya erosionadas, las tierras van desde muy superficiales hasta poco profundas con textura franco arenoso. 
Evaluación desde 0.391 a 0.846

En este tipo de zonas se considera las Cordilleras con formaciones geológicas Piloceno(relieves con pendientes fuertes al Noroeste) y Pleistoceno ( relieves volcánicos colinados muy altos-sureste) ubicadas en su mayoría dentro de páramos con pendientes entre el 25 a $40 \%$, que posee características de estructura tridimensional (granular estable, moderadamente profundo, alta porosidad), lo que produce una buena permeabilidad con alta irradiación ultravioleta que llega con gran intensidad por lo que los seres vivos que lo habitan han desarrollado estrategias de defensa como hojas peludas, hojas brillantes que ayudan a disminuir la intensidad de los rayos UV por reflexión, todo esto en conjunto permite que el páramo se convierta en una esponja para la retención e agua durante la época de sequía y durante épocas de frialdad, la materia orgánica no se descomponga.

Las zonas sin erosión se encuentran distribuidas en la parte sur y oriental del cantón y son usadas con fines de conservación y protección por tratarse de paramos arbustivos y herbáceos, un ejemplo el páramo de la Virgen en la Reserva ecológica de Cayambe

\section{Erosión del Suelo}

- Factor A, se puede observar que la erosión en la zona Figura 11. no es fuerte porque predominan valores bajos que van desde 0,000217037 a 35,95347009 (ton/ha/año) y se los representa con color verde oscuro. Mientras que los valores de erosión del suelo más altos se presentan en las zonas que tienen color rojo con un valor de 331,5691064 hasta 1018,67572 (ton/ha/año).

Un factor para la alta erosión del suelo en esta zona sería la práctica de técnicas agrícolas que se dan principalmente en la parroquia Olmedo, además si la técnica es deficiente y no permite al suelo el barbecho puede contribuir a la erosión. La mayor cantidad de erosión se ubica en suelos de tipo franco y franco arcilloso. En las zonas con escasez de vegetación, fuertes pendientes y elevadas precipitaciones, se produce fácilmente mayor erosión. 
- Factor R, la precipitación en el cantón Cayambe tiene un aumento progresivo en dirección sudeste donde la precipitación media se encuentra entre los 1241,61-1494,38 ya que se encuentra ocupando la mayor parte del área de dicho cantón.

- Factor K, en cuanto a esa gráfica se puede decir que este cantón tiene una alta susceptibilidad a la erosión encontrándose casi toda el área comprendida en los valores máximos (pon los valores de esas coloraciones) en coloración rojiza y blanca.

- Factor C, se puede decir que hay una poca pérdida de suelo en un área de cobertura y manejo específicos a un área similar, pero en barbecho continuamente labrado ya que la mayor cantidad del área se encuentra comprendida en los valores mínimos verde y amarillo pastel.

\section{$\underline{\text { Índice Ombrotérmico }}$}

De acuerdo a la Figura 13. Índice Ombrotérmico, el Cantón Cayambe posee un clima lluvioso ya que la precipitación es mayor que la temperatura, esto muestra que existen las condiciones necesarias para la existencia de vegetación. Aunque debido a varios factores que han contribuido al cambio climático, en los últimos 20 años ha incrementado la temperatura y se han observado ligeras variaciones en la intensidad y frecuencia de la precipitación.

\section{Conclusiones.}

- El cálculo de la erosión provee a los agricultores la posibilidad de tomar elección en cuanto a prácticas de cultivo y conservación del suelo, con la finalidad de que el sistema agrícola sea sostenible.

- Los factores R, L, S, K, C, A son de mucha importancia para realizar los cálculos aplicando la herramienta SIG (Algebra de Mapas). 
- El cálculo del índice ombrotérmico, calculado para periodos mensuales, ayuda con la identificación de bioclimas mediterráneos, y se puede tomar atención a la disponibilidad hídrica durante la estación cálida.

- Con el cálculo del índice ombrotérmico podemos identificar si la época es lluviosa o seca, de acuerdo a la posición geográfica del Ecuador.

- La principal amenaza climática frente a la cual se deben definir las prioridades de adaptación de los medios de vida rurales del Cantón Cayambe es la sequía, debido a que esta amenaza impacta el 100\% (6) de las parroquias y ha sido catalogada como alta.

- La segunda amenaza que impacta los medios de vida es la helada. Una de las razones por las cuales se explica que la principal amenaza climática es la sequía es la percepción generalizada de la población, quien identifica la disminución de las lluvias, que podría ser explicada, por un lado, por la irregularidad en su distribución durante el año y por otro por las características del suelo y sequedad del entorno natural.

- Se ha identificado que los principales cambios del clima en los últimos 20 años son el incremento de la temperatura y ligeras variaciones en la intensidad y frecuencia de la precipitación.

- El Déficit hídrico del Cantón Cayambe está en un rango de 236mm-316mm de precipitación, lo cual nos indica que existe un agotamiento parcial del agua disponible en el suelo, produciendo cambios en el ciclo ontogénico de la vegetación y en las condiciones atmosféricas que afectan la tasa de evaporación y transpiración dando lugar a un estrés hídrico y sumado a diversos factores determinamos que presenta suelos de tipo franco arenosos-franco arcilloso arenosos.

\section{Recomendaciones}

- Para estudios futuros, es necesario que el GAD cuente con mapa de suelos, es decir, con datos primarios para los cálculos, que permita incorporar la variable cambio climático en planes de 
ordenamiento territorial, buscando propuestas de proyectos para lograr adaptación y resiliencia de las comunidades frente al cambio climático.

- Es recomendable que se generen valores del factor $\mathrm{K}$ de acuerdo a las características del suelo de nuestro país.

- Instalar una estación meteorológica para una vigilancia a la amenaza de Déficit Hídrico.

\section{Bibliografia.}

- Díaz, J. (2014). Análisis de Erosión de suelo. Recuperado de https://www.youtube.com/watch?v=gFKHDa0dHfo

- Métodos para el estudio de la Evapotranspiración, Sánchez, MI (1992). Sociedad Española de Geomorfología.

- WorldClim. $\quad$ Recuperado $\quad 16 \quad$ de $\quad$ febrero $\quad 2016$ http://www.globalbioclimatics.org/book/bioc/global_bioclimatics-2008_09.htm

- $\begin{array}{lllll}\text { Recuperado } & 3 & \text { de } & \text { febrero } & \text { de }\end{array}$ http://www.miliarium.com/prontuario/MedioAmbiente/Atmosfera/IndicesClima.htm\#diagra ma_ombrotérmico

- Ministerio del Medio Ambiente. Recuperado el 3 de febrero de 2016 http://www.ambiente.gob.ec/wpcontent/uploads/downloads/2012/09/Documento_Metodolog+\%C2\%A1a_28_05_2012_v2_ 1.pdf

- Sanchez E, Vinueza C. (2007). Recuperado 2 de febrero de 2016 http://repositorio.utn.edu.ec/bitstream/123456789/132/7/03\%20REC\%2088\%20RESULTAD OS.pdf

- Sistema Nacional de Información. Recuperado 2 de febrero de 2016 http://app.sni.gob.ec/snilink/sni/\%23recycle/PDyOTs\%202014/0360017390001/PDyOT/28062013_155002_1.pdf

- Sistema Nacional de Información. Recuperado 2 de febrero de 2016 http://app.sni.gob.ec/snilink/sni/PDOT/ZONA7/NIVEL_DEL_PDOT_CANTONAL/LOJA/SOZORANGA/IEE/1.\% 20Documentos/Figuras/2.\%20Geopedo-log\%的\%ADa/2.2.\%20Pedolog\%C3\%ADa/Mapas/G eopedologia_Reverso.pdf

- Sistema Nacional de Información. Recuperado 2 de febrero de 2016 http://app.sni.gob.ec/snilink/sni/PDOT/ZONA2/NIVEL_DEL_PDOT_CANTONAL/PICHINCHA/CAYAMBE/IEE /MEMORIA_TECNICA/mt_cayambe_geopedologia.pdf

- INEGI. - Recuperado $\quad 3 \quad$ febrero 2016 http://www.inegi.org.mx/geo/contenidos/recnat/edafologia/doc/normedaf.pdf

- Organización de las Naciones Unidas. Recuperado 3 de febrero de 2016 https://www.oas.org/dsd/publications/Unit/oea30s/ch027.htm\#TopOfPage 
- Lianes, E. et.al. (2009). EVALUACIÓN DEL FACTOR C DE LA RUSLE PARA EL MANEJO DE COBERTURAS VEGETALES EN EL CONTROL DE LA EROSIÓN EN LA CUENCA DEL RÍO BIRRÍS, COSTA RICA. Agronomía Costarricense. 33(2). Recuperado de http://www.mag.go.cr/rev_agr/v33n02_217.pdf

- Lianes, E. (2008). ESTUDIO DEL FACTOR DE VEGETACIÓN “C” DE LA ECUACIÓN UNIVERSAL DE PÉRDIDAS DE SUELO REVISADA "RUSLE" EN LA CUENCA DEL RÍO BIRRÍS (COSTA RICA). Universidad Politécnica de Madrid. Recuperado de http://oa.upm.es/1267/1/PFC_ELENA_LIANES_REVILLA.pdf

- Estimación de la Erosión del Suelo. Recuperado de http://www.sagarpa.gob.mx/desarrolloRural/Publicaciones/Lists/CursoTaller\%20Desarrollo $\% 20 \mathrm{de} \% 20$ capacidades $\% 20$ orientadas $\% 20 \mathrm{a} /$ Attachments/23/01.pdf 